\title{
Rational Choice Theory and the Choice of Healthcare Services in the Treatment of Malaria in Nigeria
}

\author{
Dr. Bernard Owumi \\ A senior lecturer at University of Ibadan, Nigeria \\ Email: bowumi@yahoo.com \\ RAJI Sakiru O \\ A Lecturer at Houdegbe North American University, Republic of Benin \\ Email: walerajee@yahoo.co.uk
}

Accepted: Jan 17, 2013 Published: Feb 23, 2013

Doi:10.5296/jsr.v4i1.3294 URL: http://dx.doi.org/10.5296/jsr.v4i1.3294

\begin{abstract}
This paper is on the rational choice theory and the choice of healthcare services for the treatment of malaria in Nigeria. It focuses on the factors that influence or determine the choice malaria treatment using the rational choice theory as the basis. It was discovered that there were many determinants of what informs the maximum utility but it is all wrapped up in the organization of the health care system. Hence, in choosing treatment for malaria, factors like; perceived and actual quality of care, proximity of the services, accessibility, cost of treatment, socio-economic status of the patients, availability of services, etc., are important.

These factors in many occasions constitute constraints which in turn makes choices explicit and then patients makes trade-offs between alternatives.
\end{abstract}




\section{Introduction}

The fundamental unit of social life including group interaction and relationships is the individual human action. The idea of 'rational action' has generally been taken to imply a conscious social actor engaging in measured calculative schemes. It is a notable argument that human behavior is not totally spontaneous and free but determined. Rational choice is about choice based on reason. Scholar like Hechter et al, 2009, believe that any argument that takes actors and their goals as primitives or basis, and then progress towards the explanation of how the given actors' pursuit of the goals leads to systematic trends and tendencies in observed human behavior is regarded as 'rational choice'. A rational choice can be seen as one that is reasoned and also optimal for achieving a goal or solving a problem. In rational choice theories, individuals are seen as motivated by the needs, wants or goals that express their preferences. Rational behavior needs formulation of a problem that is quantifiable and measureable, and also making several strategic assumptions. Rationality factors in how much information is available and all together the formulation and background assumptions are the model within which rationality applies. Assertion of rationality without stipulating the background model assumptions describing how the problem is framed and formulated is meaningless. The rational choice method or model can be used in understanding the health seeking behavior and alternative care practices people engage in. The analysis usually begins with a question; what determines the choice of health care system of the people? Does the people's culture affect their health seeking behavioral pattern? Why would people prefer alternative medical care to the western medical care? etc. The subject matter here has to do with how people make medical choices, an important part of human behavior. How do the people choose which health care system to use, whether western or traditional and even within these sets, what subsets they decide to go for? Is life-style and behavioral risk factors influence by societal factors, or randomly distributed throughout the population without regard to social class? This paper is applied specifically to choice of health care services in the treatment of malaria in Nigeria.

For accurate application, it is important we summarize the assumptions or fundamentals of rational choice theory. Rational Choice Theory generally begins with consideration of the choice behavior of one or more individual decision-making units, which in basic economics are most often consumers and/or firms. The rational choice theorist often presumes that the individual decision-making unit in question is "typical" or "representative" of some larger group such as buyers or sellers in a particular market. Once individual behavior is established, the analysis generally moves on to examine how individual choices interact to produce outcomes. Also, the fundamental premise that the choices made by buyers and sellers (agents) are the choices that best help them achieve their objectives, given all relevant factors that are beyond their control (Obinna, 2010). The basic idea behind rational choice theory is that people do their best under prevailing circumstances.

Preferences are usually represented with a utility function where value is given to each possible alternative facing the decision maker (actor). An important result is that a preference 
relationship can be represented by a utility function only if the relationship satisfies completeness and transitivity (Onwujekwe et al., 2010). Rational choice analysis generally begins with the premise that some agent, or group of agents, is (are) maximizing utility, that is, choosing the preferred alternative.

This is only part of the story; however, another important element of the choice process is the presence of constraints. The presence of constraints makes choice necessary, and one virtue of rational choice theory is that it makes the trade-offs between alternative choices very explicit (Oladepo et al., 2008). A typical constraint in a simple one-period consumer choice problem is the budget constraint, which says that the consumer cannot spend more than his/her income. The use of utility functions means that the idea of agents making the preferred choices from among available alternatives is translated into a mathematical exercise in constrained optimization. That is, an agent is assumed to make the feasible choice (feasible in a sense that it is not prohibited by constraints) that results in the highest possible value of his or her utility function.

Another element of rational choice analysis involves assumptions about the environment in which choices are made and that is a very important aspect as we shall see later in this paper. In addition, the element of rational choice analysis is a discussion of how the choices of different agents are made consistent with one another. A situation with consistent choices in which each agent is optimizing subject to constraints is called equilibrium.

Lastly, in the absence of strong reasons to do otherwise such as the impositions like price controls by the government, the analyst employing rational choice theory will generally assume that equilibrium outcomes in the model are adequate representations of what actually happens in the real world.

\section{Malaria in Nigeria}

Malaria is a tropical infectious disease of red blood cells with protozoan parasites of the genus Plasmodium that is most prevalent in most if not all part of African countries especially Nigeria. The parasites are inoculated into the human host by a feeding female anopheline mosquito. The four Plasmodium species that infect humans are P. falciparum, P. Vivax, P. ovale and P. malaria, (World Health Organization, World Malaria Report, 2009). The first symptoms of malaria are non-specific and similar to the symptoms of a minor systemic viral illness. They comprise: headache, lassitude, fatigue, abdominal discomfort, and muscle and joint aches, usually followed by fever, chills, perspiration, anorexia, vomiting and worsening malaise. Malaria is, therefore, frequently over-diagnosed on the basis of symptoms alone, especially in endemic areas like Nigeria, because of this non-specificity of symptomatology. If treatment is not delayed and effective treatment is given, there is full rapid recovery. However, if ineffective treatment is given or if treatment is delayed, this can progress to severe malaria especially in children manifesting as coma (cerebral malaria), metabolic acidosis, severe anaemia, hypoglycaemia, acute renal failure or acute pulmonary oedema. In 
high-transmission settings like most part of Nigeria, infected but asymptomatic persons constitute an important part of the infectious reservoir. Even though treated cases (mainly children) have higher densities of gametocytes, and infectivity is positively related to gametocyte density, children constitute only a proportion of the infective reservoir. (Githeko, et al. 1992).

Studies have shown that about one million people including children, men and women die of malaria in Africa every year. (Arrow, et al, 2004, Filmer, 2005 and Ajayi et al., 2008). In the WHO African region, Nigeria accounts for $25 \%$ of all the malaria cases. Approximately $50 \%$ of the population experience at least one episode of malaria each year, while children who are aged less than 5 years (about 24 million) will have two to four attacks of malaria annually, (WHO, 2008).

Malaria is one of the major public health problems in Nigeria and its treatment is sought from a broad spectrum of public and private healthcare providers. (Onwujekwe, et al., 2005). There are many public and private healthcare providers of malaria treatment in Nigeria ranging from formal to informal healthcare providers located in urban and rural areas round the country but prompt and appropriate treatment of malaria remains a challenge in the country. This is so, considering the many potential barriers patients experiences from the first symptom of malaria to the point when the disease has been cured. Prompt and proper treatment services that are equitable and pro-poor are needed in malaria endemic countries like Nigeria in order to mitigate the impact of the disease on the poor.

A strong health system provides for effective delivery of proper treatment services to its citizens, (World Health Organization, 2007). However, in many developing countries, access to prompt and proper health services remain a major problem. Nigeria like many other Sub-Saharan African countries is bedeviled with the problem of low level of access to proper treatment especially of common illness like malaria. (Onwujekwe, Uzochukwu, et al. 2008). This is due mainly to the general poor state of the health system, high cost of health services, lopsided distribution of health facilities in favor of the urban areas and gross underfunding of the health sector resulting in lack of subsidies and exemptions to the poor. Approximately $50 \%$ of the Nigerian population experience at least one episode per year. However, official estimate suggests as much as four bouts per person per year on the average (WHO, 1995 and 2002). The trend is rapidly increasing due to the current malaria resistance to first line anti-malarial drugs (WHO, 2009). The magnitude of incidence and death due to it is a multiple of all other tropical diseases put together. It is responsible for over $90 \%$ of reported cases of tropical disease in Nigeria (Alaba and Alaba 2003). The above suggests that malaria could be the largest contributor to total disease burden and productivity losses resulting from major tropical diseases in the country. Evidence on Nigeria given by the malaria report 2005 shows that malaria incidence throughout the country had been on the increase over the years ranging between 1.12 million at the beginning of 1990 and 2.25 million by the turn of the millennium 2000 and 2.61 million in 2003. The disease carries with it two categories of costs; morbidity and mortality costs. Malaria morbidity affects households' welfare (through 
families' allocation to treatment and prevention of the disease), and decline in productivity, through lost time. In the case of mortality, losses to households include lost of future income and cumulative investment on the dead due to malaria.

\section{The Treatment of Malaria in Nigeria}

Patients in Nigeria make choices about the treatment of malaria in a diverse range of outlets in the public and private sectors. The public sector consists of primary health Centres (PHC), secondary and tertiary hospitals while the private sector is large and heterogeneous, consisting of a wide range of providers both registered and unregistered such as private hospitals, pharmacies, patent medicine dealers, medicine vendors, spiritualist and traditional healers with patent medicine dealers accounting for more than half of all providers.(Palafox, Patouillard, Tougher, et al. 2009). In Nigeria, a pharmacy is a shop where drugs are dispensed or sold and which has a qualified pharmacist attached to it. A patent medicine dealer (PMD) is one who learnt the trade of drug selling and has a shop where drugs are sold but has no professional qualifications and no pharmacist attached to the shop. A kiosk or shop is usually found in the market where people display a few drugs for sale and is usually a makeshift arrangement to suit market days, and is usually not a permanent structure. Traditional herbalists are those who use herbs or mixture of herbs to treat different ailments including malaria. All these healthcare providers offer different treatments. The hospitals would physically examine patients, sometimes ask for laboratory confirmation and then prescribe drugs for treatment. The pharmacy has a qualified pharmacist who sees to it that the correct treatment and dosage are appropriate. The patent medicine outlets are basically profit oriented and sell drugs to patients.

\section{Most Preferred Alternatives Base on Utility Function}

Many factors may determine the health-seeking behavior and choice of malaria treatment made by consumers and this should be understood for improvement in the use of appropriate treatment services. Perceived and actual quality of care administered at all levels of health care is major determinants of health outcomes and consumer's choice of treatment (Worrall, et al., 2005). In accessing the various preferences of the patients, a study in Anambra state, Nigeria, (2010), showed that the choice of treatment and the most preferred source of provision of malaria treatment services for the respondents was based on the availability of such treatment which was public hospitals followed by training of mothers, treatment in Primary healthcare centers, traditional healers and patent medicine dealers were the least preferred strategies for improving malaria treatment. Some of the preferences differed by socio-economic status and by a lesser extent, the geographical location of the patient (Uguru, et al., 2010).

The main driver for the health-seeking behavior which in turn feed the utility function is the organization of the health-care system noting that the private health sector tends to serve the 
affluent and the public sector for the poors. (Shaikh, and Hatcher, 2009). In general, the determinant factors of which treatment sources people seek are quality of service, proximity of facility, accessibility, cost of treatment, social networks and socioeconomic status of the consumers, (Uzochukwu, and Onwujekwe, 2004). Patients are usually more satisfied with drug availability and the interpersonal quality of care in private facilities due to shorter waiting times, more attentive staff and greater choice of provider (Hanson, et al, 2004).

However, studies from a number of countries noted that the technical capacity of private clinics is perceived as inferior, (Hanson, et al, 2004). According to a study carried out in Awka and Enugu-Ukwu (an urban and rural community respectively, all in the Anambra state, south-east Nigeria), it was discovered that public and private hospitals were the major providers most respondents would ideally or hypothetically prefer in the treatment of adult and childhood malaria cases. The primary healthcare is the entry point into the Nigerian healthcare sector and is aimed at providing healthcare services to the grass root; however use of primary healthcare facilities as the first resort for malaria management is low. It is estimated that only about $20 \%$ of malaria episodes are treated in the health centres, (Ajayi, et al, 2008). In order to cope with deficiencies in the performance of formal health services especially in the rural areas where malaria is most endemic, communities make the choice of an alternative means of treatment which is self-medication through the unregulated private and informal sector.

Moreover, most times they are required at these facilities to distinguish between anti-malarias and choose which one they want, (Nyamongo, 2002). Presently there are more than two hundred brands of ACTs in the Nigerian market, which can be bought over the counter, (Palafox, et al. 2009) and community members, usually, do not have appropriate information on "over-the-counter" medicines, hence this has led to widespread ineffective treatment of malaria in Nigeria, (Erhun and Osagie, 2004). The private sector is also poorly regulated, hence, the administration of anti-malarias is often erratic, (Africa Fighting Malaria (AFM), 2008). In both urban and rural areas, the first source of treatment is usually from the patent medicine dealers (PMDs), (Okeke and Okafor, 2008). Previous studies have substantiated the critical role of people's choice of PMDs in malaria treatment, indicating that they serve as the first point of contact for care in more than half of the cases in rural Nigeria, (Salako. et al. 2001, Oladepo, 2008). They are readily available and their services apparently cheaper on the short run due to nonpayment of consultation fees and transport costs, (Asenso-Okyere, et al., 1998; Hechter, et al., 2009). Public treatment services are themselves frequently inefficient, of poor quality, and underutilized and often lack drugs and diagnostic facilities, (Hechter, et al., 2009; Okonkwo, 2011). Inappropriate prescription is common in these facilities, (Goodman, et al., 2007), reducing the quality of care, wasting resources and potentially contributing to the spread of drug resistance, (Laing, et al., 2009). The poor quality of care at public facilities, crowds, long waiting times and cursory consultations, is a key factor in the preference for private providers, (Russel, 2012).

In a recent study in Swaziland, majority of the respondents stated that they would seek 
treatment within 24 hours of onset of malaria symptoms, with health facilities as their first treatment option, (Khumbulani, et al, 2011). In this study however when respondents and their children actually had malaria, most of them sought treatment at the PMDs. This finding agrees with a previous study which noted that PMDs were the choice of treatment for majority of people, (Sambo, et al, 2004). Another study also noted that the use of informal sector for malaria control is very common, (Hechter, et al., 2009). In the Sub-Saharan Africa in general, a large proportion of the drugs consumed by the people are supplied by PMDs, (Brieger, et al, 2004). Other studies in Nigeria have shown that upon recognition of malaria symptoms, most people go to PMDs for treatment as they are the easily accessible, (Onwujekwe, et al, 2008, Oladepo, et al., 2008, and Khumbulani, et al., 2011).

\section{Factors determining the choice of Malaria treatment}

From the demand side, cost of treatment is one of the major factors determining the choice of effective Malaria treatment in Nigeria (Nyamongo, 2002). Access to ACTs, is still constrained by cost as they cost much more than the previously used anti-malarias, with a dose averaging about 504 naira (\$4), (Oladepo, et al. 2008). The high cost of ACTs often results in patients opting for a cheaper alternative or not buying the full regimen, (Whitty, Allan, Wiseman, et al. 2004). This is further compounded by lack of financial risk protection in Nigeria, especially for the informal sector where the poorest people belong. This means that even the poor people pay out of pocket to receive treatment each time they have malaria which could be several times a year (Nyamongo, 2002).

From the supply side the lack of adherence to treatment guideline and poor prescribing practices of providers are major constraints to proper treatment, (Oladepo, et al. 2008). Other factors that influence provider treatment practices in other African settings include client expectations, expected profit margins, pharmaceutical company promotions, and local regulation, (Goodman, et al. 2007). Often people make an economic trade-off between either saving money on transport and buying sometimes more expensive drugs from shops or spending time and money to go to a government clinic where inexpensive or free drugs might be available, (Baume, et al., 2005). Treatment in both the public and the private sectors is hampered by low quality of care, inefficiency in service delivery and low utilization of adequate care, (Goodman, et al, 2007).

Choice is influenced by prices (including travel and time costs of seeking treatment), income, lack of information about appropriate treatment and the difficulties patients have in assessing quality treatment, (Hechter, et al, 2009). An in-depth study of PMDs in three Nigerian states revealed that relatively few of them were aware of the new government policy on anti-malaria drugs, Sulfadoxinepyrimethamine was the most common drug they stocked, followed by Chloroquine with ACTs being the least in stock and Monotherapy, artesunate drugs were also common, (Oladepo, et al, 2008). Culture also plays an important role in influencing illness recognition and choice of care, (Brieger, and Arlington, 2004). 


\section{Influence of the Environment}

However, there is a possibility that the poor and rural dwellers do not have access to appropriate treatment due to the limited number of trained healthcare providers and inequities in the distribution of these healthcare providers in favour of the urban areas, (Onwujekwe, et al, 2008). A study in Anambra state, Nigeria by Onwujekwe, et al, 2008, showed that urban-rural geographic differentials exist which determine the choice of the malaria treatment services, increasing the vulnerability of the rural dwellers to consuming inappropriate treatment which is likely to worsen their disease burden. For instance, the pattern of drug use that was found in this study has equity implications for appropriate treatment of malaria in Anambra state and even Nigeria because the two main failing drugs were used more by rural dwellers. Also, the low-level health providers with low quality of services were also significantly and predominantly used more by the rural dwellers. These findings infer that the rural dwellers received lowest quality of treatment from all ramifications and current efforts in Nigeria to provide malaria treatment services do not address geographic inequities in service delivery.

In a study by Onwujekwe et al, 2010, in Anambra State, South Eastern Nigeria using six communities- Awka, Nnewi and Onitsha (urban) and Njikoka, Aguata and Ogbani (rural) there were inequities in the quality of malaria treatment services because the better-off socio-economic status (SES) and urbanites were treated by more qualified personnel, had better diagnostic procedures and instructions from the providers compared to worse-off SES and rural dwellers. This was buttressed by the results of logistic analysis that showed a consistent positive association of SES with all perceived quality attributes.

However, in two of the logistic models, rural residence was positively associated with perceived quality of care. The rural dwellers possibly perceived higher quality of treatment because they may have lower quality expectations and so were satisfied with the treatment they chosen or received. Some authors showed that poor people bear a disproportionate burden of the disease and have poor health-seeking behaviour, thus leading them to seek treatment from "low-level" providers and avoid any form of laboratory-based formal diagnosis, (Goodman, et al., 2001; Onwujekwe, et al., 2005; Worrall, et al., 2005; Filmer, 2005), which consequently increases the burden of the disease on them, (Obinna and Onwujekwe, 2010). This means that the community setting invariably affected the socio-economic status decided to a large extent the perception of quality of treatment (utility function) and hence influenced the most preferred alternative which in turn determined the choices made. In the above study, it is seen that people were least satisfied with the services, especially diagnostic procedure and follow-up information, at Patent Medicine Dealers and Pharmacy shops, yet they are the two most common sources of treatment of malaria.

\section{Conclusion}

In conclusion, it is apparent that in choosing the health care system for Malaria, patients face 
a known set of alternatives viz, primary health care, secondary and tertiary hospitals, private hospitals, pharmacies, patent medicine dealers, medicine vendors, spiritualist and traditional healers. The preferences have axiom of completeness and the preferences are transitive in that when primary health care is preferred to patent medicine which in turn is preferred to traditional healers, then primary health care is definitely preferred to traditional healers. It is also established that patients choose the most preferred alternatives which gives the maximum utility.

There are many determinants of what informs the maximum utility but it is all wrapped up in the organization of the health care system. Hence, in choosing treatment for malaria, factors like; perceived and actual quality of care, proximity of the services, accessibility, cost of treatment, socio-economic status of the patients, availability of services, etc., are important.

These factors in many occasions constitute constraints which in turn makes choices explicit and then patients makes trade-offs between alternatives. The healthcare provider of these services are not exempted from constraints which may include lack of adherence to guidelines for treatment, client expectations, expected profit margins, pharmaceutical company promotions, local regulations, culture, etc., this means a patient may be constrained by cost of hospital treatment, thereby making his/her preference to be patent medicine store which is cheaper and still perceived as quality and relatively available. Also, a patent medicine store becomes the better option for a health service provider because of client expectation and the profit margins.

Despite the existence of these factors and constraints that determines preferences, there is also variation in the health seeking behaviour for malaria treatment of individuals in different environment. This means that there are geographical differences that influence choices of patients. It is evident from the above discussion that the way certain factors influence the decision-making in a particular environment is different from another environment. For example, in a particular geographical setting, the cost of treatment may be a constraint factor which makes patients to opt for herbal/traditional treatment and then in another environment, herbal treatment is also the option only that, it might not be as a result of the cost of treatment but maybe because of the cultural constraint on its inhabitants.

The main point here is that community settings affect the perception of the quality and organization of health care services which in turn influences the preferred alternative. This is because the environment of an individual affects his/her socio-economic status that eventually results in his/her choice of treatment. So the environment in which choices are made and many other factors are important elements in rational choice theory and it is especially important in the choice of malaria treatment.

In summary, a rational choice model for health seeking behaviour for malaria treatment can be built since preference can be laid down, maximum utility of preferences identified and choices made based on the preferences that gives more utility determined 


\section{References}

Ajayi, I. O. Falade, C. O. and Bamgboye, A. 2008. Assessment of a treatment Guideline to improve home management of malaria in children in rural south-west Nigeria. Malaria Journal. 7. 24.

Alaba, A. and Alaba, A. 2003. Consumers stated and revealed preferences for Community health workers and other strategies for the provision of timely and appropriate treatment of malaria in South east Nigeria. Malaria Journal. 5. 11.

Arrow, B. Panosian, N. and Gelbard, D. 2004. Discrete choice experiments in healthcare. Belgium Medical Journal. 2004. 328/7463. 360-1.

Asenso-Okyere, W. Anum, A. Osei-Akoto, I. and Adukonu, A. 1998. Cost Recovery in Ghana: are there any changes in health seeking behaviour? Health Policy and Planning.13.181-188.

Baume, C. Helitzer, D. and Kachur, S. P. 2005. Patterns of care for childhood Malaria in Zambia. Social Sciences Medicine. 51.10. 1491-503

Brieger, W. R. and Arlington, V. A. 2004. The role of patent medicine vendors in The management of sick children in the African region. Basic Support for Institutionalizing Child Survival: BASICS II. 126-262/29

Brieger, W. R. Osamor, P. E. Salami, K. K. and Otusanya, 2004. Interactions Between patent medicine vendors and customers in urban and rural Nigeria. Health Policy Planning.19.3.177-82.

Filmer, D. 2005. Fever and its treatment among the more and less poor in subharan Africa. Health Policy Plan. 20.6.337-46. doi:10.1093/heapol/czi043.Africa Fighting Malaria (AFM). Private sector and public sector malaria treatment in Africa: The challenge of getting the best drugs into the hands of the people who need them.

Githeko, A. K. 1992. The reservoir of Plasmodium falciparum malaria in a holoendemic area of Western Kenya. Transactions of the Royal Society of Tropical Medicine and Hygiene.86.355-358.

Goodman, C. Brieger, W. Unwin, A. Mills, A. Meek, S. and Greer, G. 2007. Medicine Sellers and Malaria Treatment in Sub-Saharan Africa: What Do They Do and How Can Their Practice Be Improved? America Journal Trop. Medicine. Hyg., 2007, 77(Suppl 6).203- 218. 
Hechter, M. Obikeze, E. N. Onwujekwe, O. E. Onoka, C. A. and Griffiths, U. K. 2009. Cost-effectiveness analysis of rapid diagnostic test, microscopy

And syndromic approach in the diagnosis of malaria in Nigeria:

implications for scaling-up deployment of ACT. Malaria Journal.8: 265.

Khumbulani, H.W. Mabaso, M. and Kunene, S. 2011. Community knowledge,

attitudes and practices (KAP) on malaria in Swaziland: a country earmarked for malaria elimination. Malaria Journal. 8:29.

Laing, R. Hogerzeil, H. and Ross-Degnan, D. 2009. Ten recommendations to Improve use of medicines in developing countries. Health Policy Plan. 16.1.13-20. doi: 10.1093/heapol/16.1.13.

Nyamongo, I. 2002. Health care switching behaviour of malaria patients in a Kenyan rural community. Social Science and Medicine, 54. 3. 377-86.

Obinna, O. Nkem, D. 1. Juliana, O. Benjamin, U. Nkoli, E. Elvis, S. 1. and Paul, O. 2006. Consumers stated and revealed preferences for community health workers and other strategies for the provision of timely and appropriate treatment of malaria in southeast Nigeria. Malaria Journal 2006, 5:117

Okeke, T. and Okafor, H. 2008. Perception and Treatment Seeking Behavior for Malaria in rural Nigeria: Implications for Control. Journal of Humanities. Ecol., 24.3. 215- 222.

Okonkwo, P. O. Akpala, C. O. Okafor, H. U. Mbah, A. U. and Nwaiwu, O. 2011. Compliance to correct dose of chloroquine in uncomplicated malaria Correlates With improvement in the condition of rural Nigerian children Trans Soc Trop Med Hyg. 95. 320-4.

Oladepo, O. Kabiru, S. Adeoye, B. W. Oshiname, F. Ofi, B. Oladepo, M. Ogungbemi, O. Brieger, W. R. Bloom, G. Peters, D. H. and Lucas, A. 2008. Malaria treatment in Nigeria: the role of patent 42. Medicine vendors. The Future Health Systems, Innovations and knowledge for future health systems for the poor. Policy Brief March.1:5 43.

Onwujekwe, O. Ojukwu, J. Uzochukwu, B. Dike, N. and Shu, E. 2005. Where do people from different socio-Economic groups receive diagnosis and treatment for malaria in southeast Nigeria. Annals of Tropical Medicine and Parasitology. 99.5.473-481.

Onwujekwe, O. Uzochukwu, B. Eze, S. Obikeze, E. Okoli, C. and Ochonma, O. 2008. Improving equity in malaria treatment: 35 . Relationship of socio- 
economic status with health seeking as well as with perceptions of ease of using the services of different providers for the treatment of malaria in Nigeria Malaria 36. Journal, 2008, 7: 5 doi:10.1186/1475-2875-7-5

Onwujekwe, O. Hanson, K. Uzochukwu, B. Ezeoke, O. Eze, S. and Dike, N. 2010. Geographic inequities in provision and utilization of malaria treatment Services in southeast Nigeria: Diagnosis, providers and drugs. Health Policy. 94: 144- 149.

Russel, S. 2012. The Economic burden of illness for households in developing countries: a review of studies focusing on malaria, tuberculosis and HIV/AIDS. American Journal of Tropical Medicine and Hygiene. 71(suppl 2):147-155.

Salako, L. 2001. Treatment of childhood fevers and other illnesses in three rural Nigerian communities. Journal of Tropical Paediatrics, 2001, 47.4.230.

Sambo, M. N. Ejenbi, C. L. and Adamu, Y. M. 2004. Out-of-pocket expenditure For under-five illnesses in a semi-urban community in Northern Nigeria. Journal of Community Medical Prim Health Care 2004;16.1.29-32.

Shaikh, B. T. and Hatcher, J. 2009. Health seeking behaviour and health service utilization in Pakistan: challenging the policy makers. Journal of Public Health 2009;27.1.49-54.

Uguru, N. P. Onwujekwe, O. E. Tasie, N. G. Uzochukwu, B. S. and Ezeoke, U. E. 2010. Do consumers' preferences for improved provision of malaria Treatment services differ by their socio economic status and geographic location? A study in southeast Nigeria. BMC Public Health 2010; 10: 7.

Uzochukwu, B. S. and Onwujekwe, O. E. 2004. Socio-economic difference and health seeking behaviour for the diagnosis and treatment of malaria: a case study of four local government areas operating the Bamako initiative programme in south-east Nigeria. International Journal of Equity Health. (6):doi:10.1186/1475-9276-3-6.

White, N. J. 2008. The role of anti-malarial drugs in eliminating malaria. Malaria Journal, 2008, 7 (Suppl 1): S8.

Whitty, C. Allan, C. Wiseman, V. Ochola, S. Nakyanzi-Mugisha, M. and Vohn, B. 2004. Averting a malaria disaster in Africa- where does the buck stop. Bulletin of the World Health Organization. 82.381- 384. 


\section{Macrothink}

Worrall, E. Suprotik, B. and Hanson, K. 2005. Is malaria a disease of poverty? A review of the literature. Tropical Medicine and International Health. 10.10.1047-1059.

World Health Organization, African Region. 2007.

World Health Organization (WHO). Malaria Report. 2009.

World Health Organization (WHO). African summit on Roll Back malaria. Abuja, Nigeria. 2000. WHO/CDS/RBM/2000.17. Geneva: WHO.

World Health Organization. 2007. Strengthening health systems to improve Health outcomes, WHOs framework for action, World 34. Health Organization, Geneva.

World Health Organization, World Malaria Report. 2009. World Health Organization

World Health Organization. 2008. African Fighting Malaria, Roll Back Malaria Partnership. Global Strategic Plan: Roll Back Malaria 2005-2015.

\section{Internet Materials}

Erhun, W. and Osagie, A. 2004. Management of Malaria by Medicine Retailers in a Nigerian Urban Community. Journal of Health \& Population in Developing Countries, 2004. URL: http:// www.jhpdc.unc.edu/. Accessed: 2/12/2011.1:45

Gilson, L. Alilio, M. and Heggenhougen, K. 1994. Community satisfaction with primary health care services: an evaluation undertaken in the Morogoro region of Tanzania. Soc Sci Med. 1994;39.6.767-80. doi: 10.1016/0277536.94.90038-8. Accessed: 2/02/2012.12:35

Hanson, K. Goodman, C. and Lines, J. 2004. The Economics of Malaria Control Interventions Global Forum for Health Research. whqlibdoc.who.int/publications/2004/2940286159.pdf. Accessed: 12/02/2012.7:55

Obinna, O. 2010. Improving quality of malaria treatment services: Assessing inequities in consumers' perceptions and providers' behaviour in Nigeria. International Journal of Equity Health. 9. 22. Published online 2010 October 11. doi: 10.1186/1475-9276-9-22 (PubMed). Accessed: 
Palafox, B. Patouillard, E. Tougher, S. Goodman, C. and Kara, H. 2009.

ACT watch: evidence for malaria medicine and policy. Outlet Survey

Report (Baseline), Nigeria 2009. www.actwatch.info/results. Accessed:

4/12/2011.8:45

www.World Health Organization Report.com. Accessed: 12/03/2012.7:15 